\title{
Temperature Activates Contact Aging in Silica Nanocontacts
}

\author{
Matthias Vorholzer, ${ }^{1, *}$ J. G. Vilhena, ${ }^{2,3,{ }^{*}}$ Ruben Perez, ${ }^{3,4}$ Enrico Gnecco, ${ }^{5}$ Dirk Dietzel, ${ }^{1,6}$ and André Schirmeisen ${ }^{1,6, \dagger}$ \\ ${ }^{1}$ Institute of Applied Physics, Justus-Liebig-Universität Giessen, 35392 Giessen, Germany \\ ${ }^{2}$ Department of Physics, University of Basel, Klingelbergstrasse 82, 4056 Basel, Switzerland \\ ${ }^{3}$ Departamento de Física Teórica de la Materia Condensada, Universidad Autónoma de Madrid, \\ E-28049 Madrid, Spain \\ ${ }^{4}$ Condensed Matter Physics Center (IFIMAC), Universidad Autónoma de Madrid, E-28049 Madrid, Spain \\ ${ }^{5}$ Otto Schott Institute of Materials Research, Friedrich Schiller University Jena, 07742 Jena, Germany \\ ${ }^{6}$ Center for Materials Research, Justus-Liebig-Universität Giessen, 35392 Giessen, Germany
}

(Received 24 April 2019; revised manuscript received 16 September 2019; published 2 December 2019)

\begin{abstract}
Understanding the time evolution of contact strength in silica nanocontacts is of great fundamental and practical relevance in diverse areas like earthquake dynamics, wafer bonding mechanisms, as well as MEMS applications. The logarithmic increase of contact strength with hold time, termed contact aging, can be "quantitative" due to deformation creep in plastic contacts. An alternative mechanism, termed "qualitative aging," is the gradual change in interfacial chemistry, which so far was only observed in the presence of humidity. Here we present nanoscale friction experiments of dry silica contacts in ultrahigh vacuum that show a doubling of shear strength with time following a logarithmic law. We find that the aging rate scales linearly with temperature, and that shear stress shifts the relevant energy barriers. All-atom MD simulations provide a live picture of the bond formation dynamics occurring at the interface. Our experiments link contact aging to thermally activated bond formation, show that it exists even in the absence of water molecules, and demonstrate that this atomic aging mechanism can stretch over timescales up to several seconds. Qualitative contact aging is thus highly relevant for a broad variety of material combinations and conditions.
\end{abstract}

DOI: 10.1103/PhysRevX.9.041045

Subject Areas: Computational Physics, Materials Science

\section{INTRODUCTION}

Around 350 A.D. the Greek philosopher Themistius reported that [1] "One may more easily further the motion of a moving slider than to move a body at rest." In spite of the ubiquity of this observation, understanding the origins of static friction is a challenging task and therefore the subject of vibrant research [2-9]. The complexity of the process can be best understood starting from the idea that virtually any macroscopic surface exhibits a significant roughness, leading to a certain number of true contact points at the interface $[10,11]$. The dynamics of the junctions is then governed by a broad range of complex physical-chemical processes including bond formation [12-16], plastic flow [17], capillarity [18,19], and atomic attrition [20]. Through the time-dependent nature of these processes the contact

\footnotetext{
*These authors contributed equally to this work.

†schirmeisen@uni-giessen.de
}

Published by the American Physical Society under the terms of the Creative Commons Attribution 4.0 International license. Further distribution of this work must maintain attribution to the author(s) and the published article's title, journal citation, and DOI. strength is expected to increase logarithmically with time. Indeed, it has been shown that the static friction increases logarithmically with the hold time [3,21], which is usually described in the rate-and-state models. However, an atomistic understanding of this phenomenological "law" is still missing.

In order to understand the atomic origins of aging, recent work has focused on silica contacts, which are relevant in a huge variety of technical and environmental systems [22-24]. It was shown that aging can be categorized into two different phenomena: quantitative and qualitative aging. The former, an established and widely accepted mechanism, describes the time-dependent increase of the real contact area due to plastic deformation and material creep [21,25]. More recently, researchers have focused on the so-called qualitative aging, i.e., the change of the contact strength per unit area usually controlled by the interfacial chemistry. This has spurred experiments based on friction force microscopy (FFM), where a single asperity contact geometry ensures a fixed contact area and any sign of aging should be dominated by qualitative changes of the interface. In fact, strong aging effects have been discovered with FFM, in particular for hydrophilic silica surfaces under ambient conditions [3]. Ab initio simulations have 
confirmed that covalent bond formation, i.e., the formation of siloxane bridges, can lead to the observed logarithmic increase of contact strength with time [12].

If such a multibond formation process is relevant for contact aging, it should play an important role in any contact where chemical bond formation is feasible. In particular, this should hold for atomically clean surfaces, where bond formation is not inhibited by passivating layers. Contact aging effects were indeed observed on a clean metal surface in ultrahigh vacuum (UHV) at cryogenic temperatures [8]; however, in metals bond formation and material creep are so intimately connected that is almost impossible to disentangle those two mechanisms. Logarithmic contact aging has likewise been observed for metal nanoparticles sliding on graphite [26], but in that case atomic bond formation between $\mathrm{Sb}$ and graphite is impossible and aging is due to atomic creep mechanisms. FFM experiments on $\mathrm{NaCl}$ surfaces also resulted in frictional strengthening, but here also due to creep caused by atomic attrition mechanisms [20].

Another aspect of aging due to multiatom bond formation is that those processes are inherently thermally activated. Temperature should thus strongly influence the aging dynamics and, indeed, theory predicts that the aging rate scales linearly with temperature [12], which, however, lacks experimental confirmation. Also, according to Eyring's law, the energy barriers responsible for bond formation can be significantly reduced by shear stress, leading to a load dependence of aging. Recent experiments for silica contacts in ambient conditions could not confirm this, but instead found "that the average pressure, and thus the average bond formation rate, was load independent within the accessible load range" [14]. This leaves open the question of if and how strongly chemical bond formation influences contact aging.

The most direct route to validate chemical-bond-based contact aging is thus to measure the temperature dependence of aging rates of atomically clean single asperity model interfaces. Indeed the friction of clean silica-silica contacts in UHV have been successfully described by a multibond formation process and might provide an excellent sample system [15]. However, recent slide-hold-slide experiments of such a system completely failed to observe contact aging at room temperature [27]. One reason may be that atomic bond formation for silica bonds proceeds too quickly in this case, as suggested by previous friction experiments [15]. Only at low, cryogenic temperatures bond formation rates could slow down such that they fall into experimentally accessible timescales.

At room temperature, current research indicates that qualitative contact aging for silica scales with humidity and is irrelevant in dry environments. For rock friction this would mean that in the absence of water, which is expected to be the case in seismic faults, the shear stress per real unit area remains constant, with corresponding consequence for earthquake simulations in the frame of rate-and-state models. Equally, under these assumptions, for wafer bonding processes humid and ambient environments must be considered crucial to facilitate the gradual bond formation across the interface, while using dry or vacuum conditions might be regarded as a viable route to prevent aging and wear in silicon-based microelectromechanical systems (MEMS).

Here we directly challenge the broadly accepted assumption on the absence of aging in dry $\mathrm{SiO}_{2}$ nanocontacts through friction force microscopy experiments of dry silica nanocontacts in ultrahigh vacuum conditions. We demonstrate that the conventional FFM-based static friction measurement protocol is inadequate to observe contact aging, further rationalizing previous failure to observe this effect. Instead, we show that the time evolution of the contact stiffness gives clear evidence of qualitative contact aging in agreement with the thermally activated atom bond formation picture, including the predicted scaling of the aging rate. Moreover, we find an Eyring-type shift of the energy barriers due to shear stress. Complementary allatom MD simulations of silica nanocontacts corroborate the experimental results.

\section{RESULTS AND DISCUSSION}

In our experiments we used temperature-dependent friction force microscopy [28] to measure the lateral forces at the interface between an AFM cantilever covered by $\mathrm{SiO}_{2}$ and $p$-type (111) $\mathrm{Si}$ wafers with either a native oxide layer or a 300-nm oxide layer. Since humidity is known to have a strong influence on microscopic and macroscopic contact aging [12,13,29], all experiments were conducted under ultrahigh vacuum conditions.

The measurement protocol was inspired by the slidehold-slide procedure introduced by Li et al. in their seminal work on this topic [3]. Essentially, the tip is elastically pulled laterally along the substrate, then halted for a welldefined time $t_{\text {hold }}$, after which the pulling process is restarted [Fig. 1(a)]. The sliding friction $F_{\text {sliding }}$ can be derived from the average value of the force profile in the first sliding phase [corresponding to the green region in Fig. 1(b)]. After a sudden drop, which is related to cantilever creep during the hold time, the lateral force increases almost linearly when the cantilever is pulled again along the surface. In this hold phase the tip is effectively pinned to the sample, and once sliding is reinitiated, a force constant can be calculated from the slope of the force profile which corresponds to the effective spring constant $k$ of the pinned contact region combined with the AFM cantilever under torsion. The cantilever creep is related to the close match between cantilever torsion and friction force but is effectively limited to the first few milliseconds of the hold time (see the Appendix B) and is thus not significant for the general interpretation of the slide-holdslide process where the contact can still be assumed as 

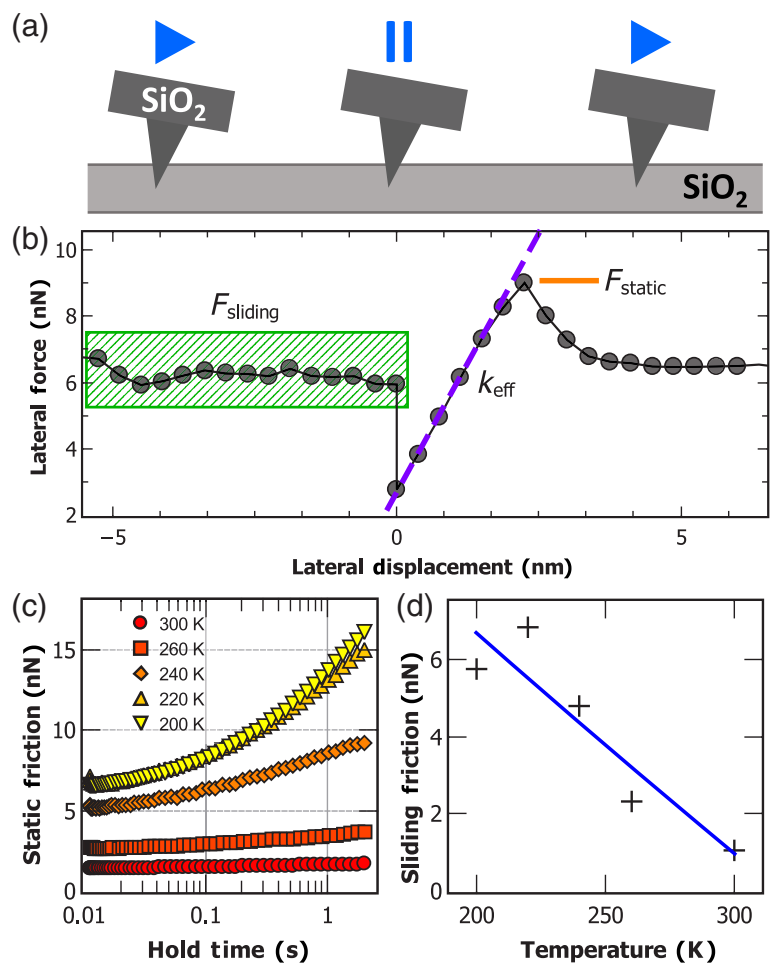

FIG. 1. Slide-hold-slide experiments performed on $\mathrm{SiO}_{2}$. (a) Schematic course of a typical slide-hold-slide procedure, where tip movement is paused for a defined amount of time. (b) Exemplary slide-hold-slide curve obtained for silica on native silica at $200 \mathrm{~K}$ with $t_{\text {hold }}=2.1 \mathrm{~s}$. The $x$ axis is relative to the stopping point. As indicated in the figure, various characteristic quantities are evaluated around the stopping point. Especially important are the sliding friction level $F_{\text {sliding }}$ (green), the static friction peak $F_{\text {static }}$ (orange), and the contact stiffness $k$, which can be derived from the slope of the lateral force buildup (purple, extrapolated as dashed line). (c) Hold time dependence of the static friction for different temperatures between $200 \mathrm{~K}$ (yellow) and $300 \mathrm{~K}$ (red) measured on the native oxide layer. (d) Temperature dependence of the sliding friction $(v=2.5 \mu \mathrm{m} / \mathrm{s})$ for the native oxide silica (crosses) fitted by a theoretical curve (blue line) based on the standard model describing thermally activated friction.

static. The instant at which the contact is broken corresponds to a maximum value defining the static friction $F_{\text {static }}$. At this point, the tip motion is resumed and the lateral force starts its decay toward the initial sliding friction value, which occurs within a certain transient time. Altogether, the force variations were recorded along a path of $300 \mathrm{~nm}$ and the measurements were repeated between 160 and $350 \mathrm{~K}$.

As a first result, we observe that both the static friction and the sliding friction decrease with temperature $T$ [see Figs. 1(c) and 1(d)] in line with the well-known picture of thermally activated slip originally proposed by Prandtl for atomic-scale sliding friction [30-32]. In this model thermal fluctuations lead to a premature slip of the contact, which would otherwise occur when the theoretical shear strength of the interface at zero temperature is reached. The higher the temperature, the larger the amplitude of these fluctuations, which consequently results in a decrease of the friction force, which was demonstrated experimentally [32]. Our analysis shows that the static friction increases with contact time for all temperatures, in line with the contact aging models, but the increase is more pronounced than a simple logarithmic behavior. Even more puzzling is that the growth rate of the static friction decreases with increasing temperatures. This is in direct contradiction with the principles of thermally activated bond formation at the interface, which should be activated by temperature.

The reason for this apparent paradox is related to the dynamic nature of the static friction peak. So far, this peak in slide-hold-slide experiments has been assumed to be proportional to the number of interfacial bonds [3,12,14,27]. This is problematic, since any static friction measurement is a complex process including contact rupture dynamics on top of the aging process itself, both of which are characterized by distinct temperature dependencies. The result is a highly complex dynamic process where static friction is not a direct measure of the number of interfacial bonds. This is particularly relevant for nanoscale contacts, where bond rupturing can be significantly thermally activated $[15,26,33]$, and it becomes especially apparent if experiments at different temperatures are compared, as is our case. This also explains why recent attempts to measure static friction of dry silica contacts have proven to be not so conclusive [27]. At room temperature, bond rupturing is strongly thermally activated $[15,20,26]$, basically burying the dynamics of the bond formation process and making it difficult to observe qualitative aging.

A more direct measure of the number of atomic bonds is the lateral contact stiffness $k$. Figure 2 shows the dependence of $k$ on the hold time $t_{\text {hold }}$, extracted from the same experiments. The minor slope decrease preceding the slip is well known from the theory of atomic-scale and is caused by the slow onset of the tip slip [34]. For this reason, $k$ is better estimated from the increase of the lateral force built up immediately after the hold phase, when the tip starts to be pulled again. If we analyze only forces well below the rupture force, the interface remains in a quasistatic state and the initial contact stiffness is a good measure for the interface condition after $t_{\text {hold }}$. In an atomic bond picture, the aging of silica contacts is described by the time evolution of the number of atomic bonds at the resting interface $[12,13]$. Assuming that each interfacial bond corresponds to a nanospring with average spring constant $k_{1}$ along the pull direction, a parallel arrangement of bonds will simply result in an effective stiffness $N k_{1}$; i.e., it will be proportional to $N$. A logarithmic increase of the number of interfacial bonds, $N(t, T)=N_{0}(T)+\alpha(T) \ln \left(t / t_{0}\right)$, should then result in an increase of the effective contact stiffness, $k(t, T)=N(t, T) k_{1}$. Indeed, a very regular logarithmic behavior of $k$ with $t_{\text {hold }}$ is observed on the native oxide layer [Fig. 2(a)], as well as on the 300-nm thin silica film 

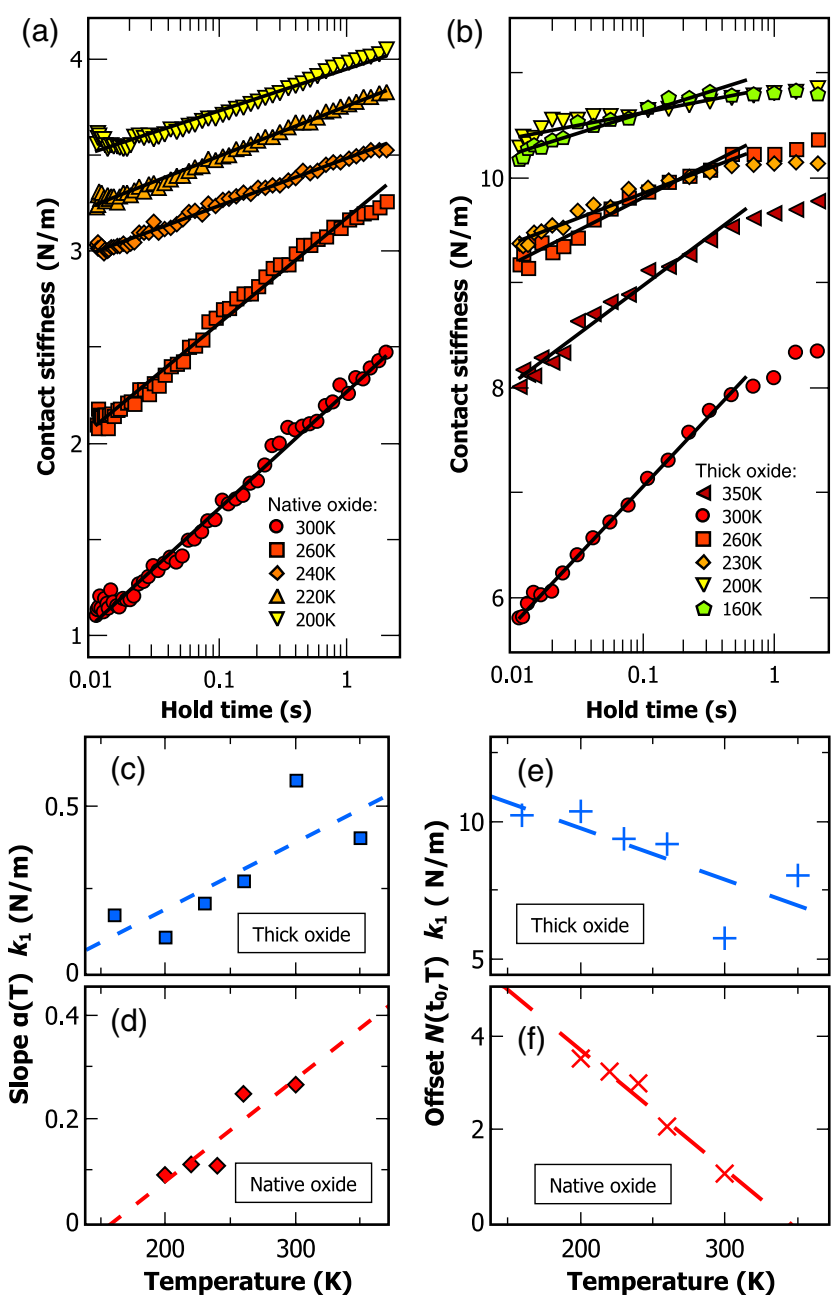

FIG. 2. Characterizing contact aging dynamics by contact stiffness measurements. Contact stiffness as a function of hold time measured for different temperatures on silicon with a native oxide layer (a) and a 300-nm-thick oxide layer (b). The contact stiffness shows logarithmic aging, and fits (solid black lines) for hold times up to $t_{\text {hold }}=0.6 \mathrm{~s}$ reveal temperature-dependent slopes, as shown for the native oxide layer (c) and the 300$\mathrm{nm}$-thick oxide layer (d). In both cases, the temperature dependence of the slopes can be well approximated by a linear function with almost identical slope. (e),(f) Initial contact stiffness at $t=10 \mathrm{~ms}$ as a function of the temperature. In both cases, the initial contact stiffness decreases with temperature.

[Fig. 2(b)]. In both cases we find that higher temperature results in a faster logarithmic increase of $k$ with the contact time [Figs. 2(c) and 2(d)]. In particular, the aging rate $\alpha k_{1}$ scales linearly with temperature. Interestingly, the observed logarithmic behavior is also expected [35] for any two-level process, i.e., for bond formation or rupture. More specifically, a simplified analytical model proposed by Liu and Szlufarska [12], based on thermally activated bond formation, predicts that $\alpha$ is directly proportional to temperature with a constant prefactor, i.e., $\alpha(T)=\alpha_{0} T$, in agreement with our findings. At last, since both native and thick oxide surfaces behave similarly, this provides further evidence that only the upper atomic layers are involved in the aging process, as expected for the thermally activated bond formation aging mechanism and in line with previous simulations $[12,13,36]$. Note that the absolute values of contact stiffness in Figs. 2(a) and 2(b) cannot be compared directly, as two different tips were used in the two sets of experiments.

Despite Figs. 1 and 2, to our knowledge constituting the first experimental evidence for thermally activated aging in dry silica contacts, there is a wealth of experiments [37-39] and simulation [12,13,36,37,40-42] works focused on stress-induced bond formation in $a-\mathrm{SiO}_{2}$, indirectly supporting our findings. Namely, several MD simulations $[12,13,36]$ predict that the indentation process results in siloxane bond (i.e., $\mathrm{Si}-\mathrm{O}-\mathrm{Si}$ ) formation between tip and surface, which was also confirmed by our MD simulations; see Fig. 3(a) cyan inset. Interestingly, aberration-corrected transmission electron microscopy [37], pair distribution function analysis of 2D x ray [38], and NMR spectroscopy [43] revealed the soft nature of this bond. In fact, these works conclusively [38,40-43] demonstrate the importance of bond angle change (of $\mathrm{Si}-\mathrm{O}-\mathrm{Si}$ or $\mathrm{O}-\mathrm{Si}-\mathrm{O}$ ) versus bond stretching for strain accommodation in amorphous silica. Therefore, in our experiments, once the siloxane bond between tip and surface has been formed, the deformation process resulting from the slide-hold-slide protocol will first cause and angle distortion of the $\mathrm{SiO}_{4}$ tetrahedron, as schematically represented in Fig. 3(a) gray inset. It would be interesting to compare this soft deformation, with a torsional stiffness $k_{\theta}$ in the order of $10^{-19} \mathrm{~J} / \mathrm{rad}^{2}$ [44], corresponding to a lateral stiffness $k_{1}=$ $k_{\theta} / R^{2} \sim 1 \mathrm{~N} / \mathrm{m}$ (where $R=0.35 \mathrm{~nm}$ is the length of an unstretched siloxane bridge), to the values of $k$ presented in Fig. 2. Although the initial values at high temperature $(300 \mathrm{~K})$ are in the same order of $k_{1}$, a direct comparison is not possible without taking into account the complex network of chemical bonds (here represented as springs) of the contacting objects in the proximity of the contact area. In particular, it would be wrong to estimate the number of contact points in the experiment simply as $N=k / k_{1}$. It would mean that the contacting springs are rigidly arranged in parallel, but this brutal assumption underestimates $N$. A detailed analysis of this problem is left for future work.

Building on the success of $\mathrm{MD}$ in describing the mechanical properties of $a-\mathrm{SiO}_{2}[37,38,40-43]$ while providing the enlightening atomic-scale picture of such processes, here we follow these works in order to obtain the atomic detail of the thermal activated contact aging process. We considered the indentation process of a realistically large $a-\mathrm{SiO}_{2}$ tip or surface composed by 352137 atoms with an experimentally matched roughness [45] and let them evolve under a constant loading force $F_{N}$ for long periods of time (see Appendixes C, D, and E and Fig. 3 for details). Although the well-known limitations of fully atomistic methods applied to tribological processes $[12,13,46-48]$ 

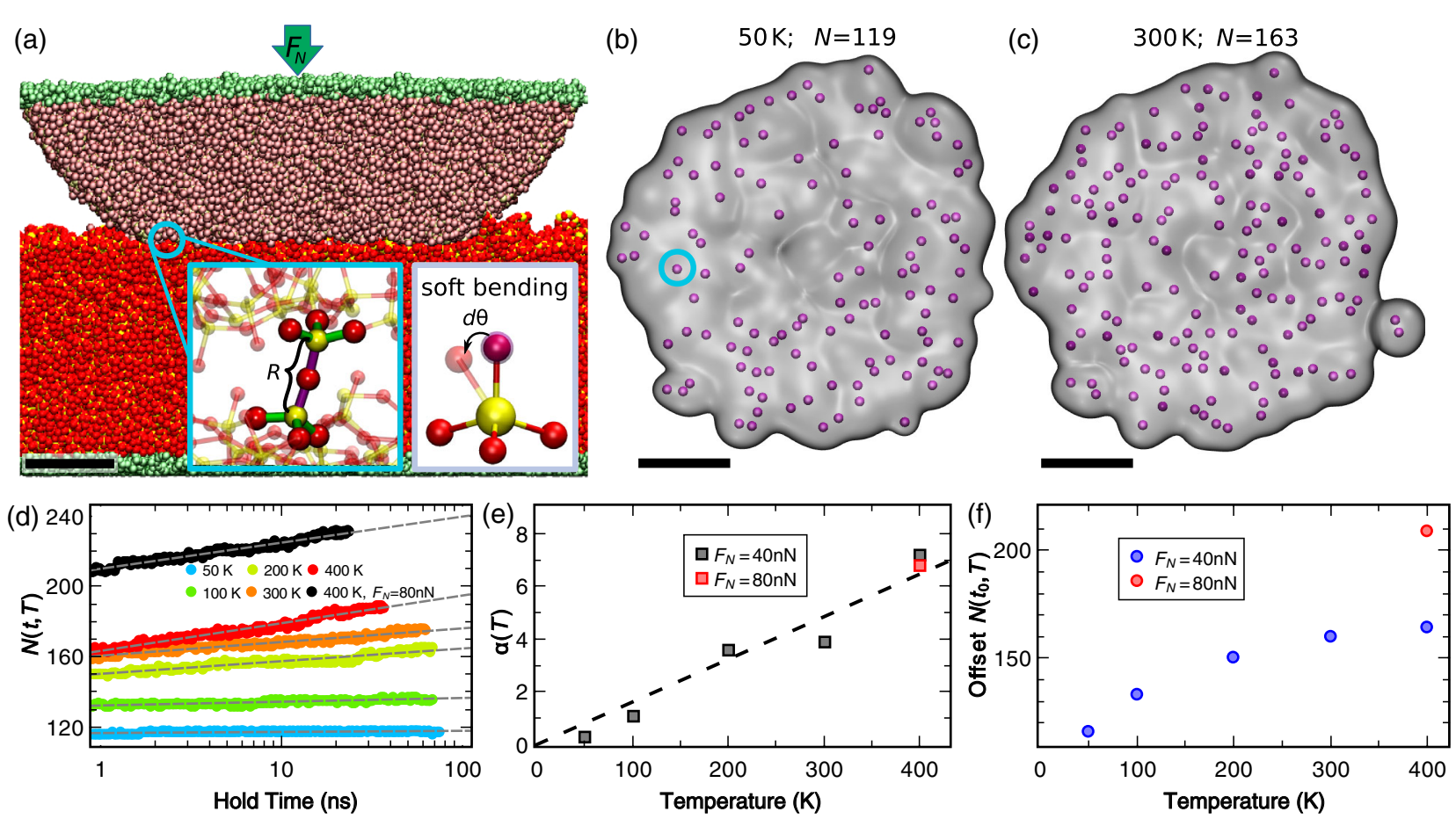

FIG. 3. MD simulations of $\mathrm{SiO}_{2}$ contact aging under an applied normal load $F_{N}$. Tip-surface atomic representation after (a) 75 ns of indentation in the MD simulation performed at $50 \mathrm{~K}$ with a normal load of $F_{N}=40 \mathrm{nN}$. The $\mathrm{O}$ and $\mathrm{Si}$ atoms are, respectively, represented in red and yellow van der Waals spheres. The insets in (a) correspond to a representative siloxane bridge, i.e., Si-O-Si, between the tip and surface (cyan box) and a representation of a soft [44] angle deformation in such contact that could account for the small contact stiffness measured. (b),(c) In purple we represent the atoms from the tip bonded to surface atoms after $70 \mathrm{~ns}$ of simulation at two different temperatures, i.e., 50 and $300 \mathrm{~K}$. The corresponding temperatures and number of bonds $(N)$ are shown. With a gray surface we represent the area of the tip at a distance smaller than $3 \AA$ from the surface. (d) Logarithmic time evolution of the total number of bonds- $N(t, T)$ - between the tip and the surface obtained at different temperatures $T$. Here we are considering in the count both $\mathrm{Si}-\mathrm{O}$ and $\mathrm{O}-\mathrm{O}$ bonds; for further details on the criteria for bond formation, see Appendix E. All curves were obtained from simulations performed at $F_{N}=40 \mathrm{nN}$, with exception of the black curve where we used $F_{N}=80 \mathrm{nN}$. In dark gray we represent the logarithmic fit, i.e., $N(t, T)=\alpha(T) \ln \left(t / t_{0}\right)+N_{0}(T)$, to each of the datasets. (e) Temperature dependence of the slopes, i.e., $\alpha(T)$, describing the temporal evolution of the bonds formed in the contact as shown in (c). Note that for $F_{N}=80 \mathrm{nN}$ we represented the data point in coral. (f) Temperature dependence of the initial bond concentrations, i.e., $N\left(t_{0}=1 \mathrm{~ns}, T\right)$. Again, the data point obtained for $F_{N}=80 \mathrm{nN}$ is represented in coral. The scale bar in all images corresponds to a distance of $3 \mathrm{~nm}$.

restrict us to much smaller timescales than experimental ones, these are still long enough to appreciate the logarithmic time dependence of the bond formation process expected from the experimental results [12,13]. The results in Fig. 3(d) show the number $N$ of bonds formed between tip and surface as a function of the contact time $t$. In the cyan boxed inset of Fig. 3(a) we show one representative siloxane bond occurring at the tip-surface interface. For all temperatures $T$ considered, $N(t, T)$ increases logarithmically with time as $N_{0}(T)+\alpha(T) \ln \left(t / t_{0}\right)$, where $\alpha$ represents the aging rate and $N_{0}(T)$ corresponds to the atomic bonds formed before the logarithmic aging is observed [see Fig. 3(d) and Figs. 3(b) and 3(c) for spacial distribution of these bonds at different temperatures]. More importantly, as shown in Fig. 3(e), the slope $\alpha$ is found to increase linearly with $T$ in agreement with our experimental observations of the contact stiffness. Therefore, MD simulations not only support that contact aging occurring in dry silica nanocontacts emerges from the gradual formation of siloxane at the contact, in agreement with previous works $[12,13,36]$, but also that an increasing temperature results in a faster aging (bond formation) rate, as shown in Figs. 3(b) and 3(c). Furthermore, quite surprisingly the complex bond dynamics occurring at the interface of such large contact is well described by the simple analytical model by Liu and coworkers [12,13] based on drastic assumptions, such as a homogeneous distribution of energy barriers in a welldefined energy interval ranging from 0 to $E_{0}$. Indeed, in the intervals between 20 and 80 ns that are here considered, bonds are repeatedly formed and broken at different sites in the contact region, with a complex interdependent energy barrier distribution. Therefore, both our simulations and experiments provide support for the nontrivial linear temperature dependence of $\alpha$ proposed by Liu and Szlufarska [12] and validate the conclusions of this simplified model well beyond its restrictive assumptions. Furthermore, the very similar rates obtained at different loads $F_{N}=40$ and $80 \mathrm{nN}$, see Fig. 3(e), indicate that the 
aging rate does not depend on $F_{N}$ [whereas the initial contact area or number of bonds is considerably larger when the load is increased, see Fig. 3(f)].

Finally, we address the observation that in FFM measurements the initial value of the contact stiffness decreases with the temperature, as shown in Figs. 2(e) and 2(f). According to the aforementioned atomic bond picture, this seems to imply that the higher the temperature, the lower the number of initial bonds in the contact. Given that bond formation is a thermally activated process, this result looks, at first, surprising. In fact, our MD simulations at $F_{N}=$ $40 \mathrm{nN}$ predict a small increase in the initial number of bonds with the temperature ( $\sim 7 \%$ in the range of temperatures used in the experiments). Nevertheless, it can be understood by considering how the contact is prepared experimentally. Since the tip is halted while sliding, the interface starts aging under finite shear stress conditions which are highly temperature dependent. As shown in Fig. 1(d), the sliding friction decreases with increasing temperature. As a result, the higher the temperature, the lower the shear stress in the contact. Comparing the MD simulation at different loads we observe a similar behavior: i.e., lower loads, or equivalently lower contact stress, correspond to a smaller initial number of bonds [see Fig. 3(f)] that result in a smaller initial contact stiffness, in agreement with the experiments. Most importantly, the simulations clearly show that the rate at which the number of bonds increases with the temperature, i.e., $\alpha$, does not depend on the contact stress [see Fig. 3(e)] for the range of loads employed here. Moreover, these findings are in agreement with Eyring's law [49] according to which this stress shifts the energy barrier distribution for bond formation, a mechanism that was also confirmed to apply for nanocontacts in several previous studies [14,50]. In our case, the shear stress allows us to form a larger number of bonds during the first $10 \mathrm{~ms}$ of hold time, while the subsequent bond formation rate related to bonds of higher energy barriers remains unchanged by the shear stress.

Overall, our findings highlight not only the importance of qualitative aging (contact strength) with respect to quantitative aging (contact area), but also shed light on its atomic origins. Guided by its atomic origins, one may expect that our results go well beyond the case of simple silica contacts. In fact, it is sensible to assume that in any homocontact of covalently bonded materials (e.g., diamondlike carbon, $\mathrm{SiC}$, etc.) the naturally present dangling bonds, defects, and step edges naturally present will provide a route to a chemically induced qualitative contact aging analogous to $\mathrm{SiO}_{2}$ contacts. It is worth mentioning the recent experiments by Weber et al. [51] that revealed a surprising increase of the real contact area [52,53] (quantitative aging) during macroscopic slip events, which are accompanied by a reduction in friction. Then it follows that the smaller shear stress per unit area (qualitative aging) - directly related to the contact stiffness shown in
Fig. 2-underpins the weakening of dynamic friction with respect to the static one. This suggests that a synergetic combination of single asperity slide-hold-slide experiments with real contact area measurements [51-53] allows us to further explore the thus far elusive qualitative aging from idealized to realistic conditions, thus providing new insight into our understanding of third-body chemistry and how this process governs aging processes at nanoscales and, consequently, macroscales.

\section{CONCLUSION}

In summary, the temperature-dependent measurements presented in this paper show that contact aging of silica interfaces in the absence of water is observed on the timescale of up to several seconds, and is a thermally activated process. This is demonstrated by first noticing that the lateral contact stiffness $k$ is a more suitable marker of contact aging, as compared to the static friction. Being proportional to the number of interfacial bonds, $k$ scales logarithmically with the contact time, and its rate scales linearly with temperature, in full agreement with the accompanying MD simulations of the contact evolution. Our results also shed light on the distinction between contact "quantity" and "quality," which has been recently proposed in the literature. The average number of interfacial bonds seems to be all that is needed to characterize the qualitative aging of a contact between two surfaces in dry conditions. Finally, our experiments also highlight that faster contact aging does not necessarily lead to a faster increase of static friction, an effect which can be attributed to the often overlooked role of rupture in static friction measurements.

\section{ACKNOWLEDGMENTS}

D. D. and A.S. are thankful for financial support provided by the German Research Foundation (Projects No. DI917/6-1, No. DI917/7-1, and No. SCHI619/10-1). R. P. and J. G. V. are thankful for the financial support of the Spanish MINECO (Projects No. MAT2014-54484-P and No. MAT2017-83273-R) and also the computer resources, technical expertise, and assistance provided by the Red Española de Supercomputación at the Minotauro Supercomputer (BSC, Barcelona) and the Extremadura Research Centre for Advanced Technologies (CETACIEMAT). J. G. V. acknowledges funding from a Marie Sklodowska-Curie Fellowship (Grant No. DLV-795286) within the Horizons 2020 framework. All authors acknowledge the financial support provided by the COST Action MP1303.

\section{APPENDIX A: EXPERIMENTAL METHODS}

All low-temperature AFM experiments have been performed using a standard Omicron VT-AFM system at pressures of $p \leq 5 \times 10^{-10} \mathrm{mbar}$ and $\mathrm{Si}$ cantilevers with a normal force constant of $k=0.2 \mathrm{~N} / \mathrm{m}$ (Nanosensors, 
PPP-LFMR). The lateral forces were calibrated using the calibration technique introduced by Bilas et al. [54]. To remove residual water and hydrocarbons, all tips and samples have been cleaned by thermal treatment at a temperature of about $250^{\circ} \mathrm{C}$ for $3 \mathrm{~h}$ under UHV conditions prior to the AFM measurements.

Our contact aging measurements are based on an experimental approach similar to the slide-hold-slide protocol introduced by Li et al. [3]. We recorded conventional friction loops of $300 \mathrm{~nm}$ width, where scanning was suspended for a defined time $t_{\text {hold }}$ in the middle between the two cantilever turning points to initiate contact aging. For both static and sliding friction, the required reference level of the lateral force signal is extracted from the full friction loops. A relatively high scan speed of $2.5 \mu \mathrm{m} / \mathrm{s}$ has been used throughout all of our experiments, primarily to minimize effects of gradual and thermally activated contact breaking. For measurements with systematic variation of the hold time at a constant interface temperature, one line of $300 \mathrm{~nm}$ width was repeatedly scanned with a nominal normal load of $1.2 \mathrm{nN}$ while varying the hold time. Starting from $t_{\text {hold }}=10 \mathrm{~ms}$ the hold time was steadily increased up to $2.5 \mathrm{~s}$ and subsequently decreased back to $10 \mathrm{~ms}$. No significant differences were found between these two ramps. After each cycle in hold time, the tip was moved to a new sample area and the procedure was repeated, resulting in 40 positions each measured for 20 hold times between $10 \mathrm{~ms}$ and $2.5 \mathrm{~s}$.

In the slide-hold-slide experiments, the effective contact stiffness $k$ between the tip and the sample is evaluated from the slope of the lateral force buildup that occurs directly when sliding is reinitiated. Only the first $30 \%$ of total height of any friction peak are used for contact stiffness determination to avoid including data points already within the onset of rupture.

Before and after the slide-hold-slide experiments, conventional AFM images larger than the sample area are taken to ascertain a clean surface area and to evaluate the occurrence of any alterations induced by the experiment. Temperatures below 150-200 K occasionally showed wear and were thus altogether excluded from the contact stiffness evaluation. To ensure that no systematic influences related to tip degradation affect the experiments, the temperature sequence has been randomized, and frequent reference point measurements at room temperature turned out to be highly reproducible.

\section{APPENDIX B: CANTILEVER CREEP EFFECTS}

In Fig. 1, a sharp decrease of the lateral force was observed at $x=0$, i.e., after the hold time. This observation is universal for all slide-hold-slide curves and can be attributed to a creeping relaxation movement of the tip during the hold time. This creep motion becomes understandable, when considering that the cantilever torsion measured as sliding friction is exactly the force to sustain cantilever sliding. This

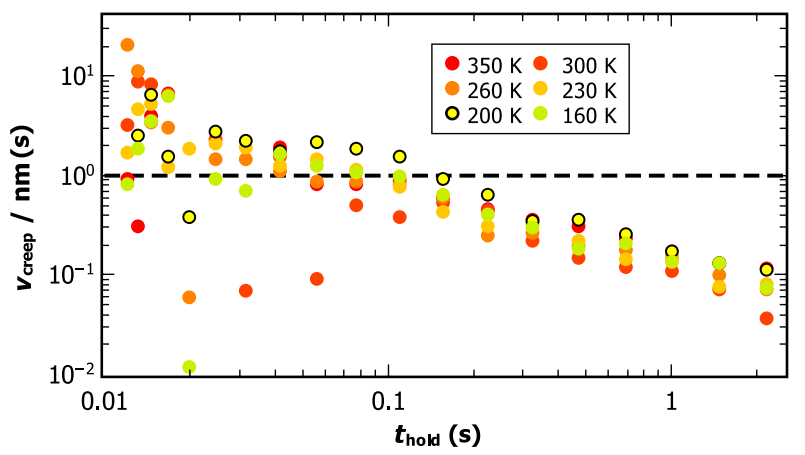

FIG. 4. Temperature-dependent creep velocities $v_{\text {creep }}$ as a function of the hold time $t_{\text {hold }}$ obtained for a typical set of slide-hold-slide experiments.

means that when the tip is halted, a lateral force equal to the sliding friction initially acts on the interface between tip and sample. This force, in combination with thermal activation, leads to a motion of the tip in scanning direction and accordingly to a reduction of the shear stress at the interface with time. From Eq. (B1), the time-dependent velocity of this creep motion can be estimated based on the lateral force values $F_{\min }$ recorded directly after each hold time:

$$
v_{\text {creep }}=\frac{1}{k_{x}} \frac{F_{\min , i}-F_{\min , i-1}}{t_{\text {hold }, i}-t_{\text {hold }, i-1}},
$$

where $F_{\min , i}$ and $F_{\min , i-1}$ are the minimum lateral forces after the hold times $t_{\text {hold }, i}$ and $t_{\text {hold }, i-1}$, respectively. Additionally, $k_{x}$ denotes the effective lateral stiffness of the cantilever.

The resulting creep velocities as a function of the hold time are shown in Fig. 4 for a typical set of slide-hold-slide experiments and realistic values of $k_{x}$. Velocities down to the picometer per second range can be resolved by this approach. A finite velocity is observed at any given hold time, yet the velocity rapidly decreases with time. After $50 \mathrm{~ms}$, the creep velocity has already dropped to approximately $1 \mathrm{~nm} / \mathrm{s}$, which is 3 orders of magnitude slower than the sliding velocity. For the example shown in Fig. 4, the total slip distances during the maximum hold time of $2 \mathrm{~s}$ vary between $0.7 \mathrm{~nm}(300 \mathrm{~K})$ and $1.4 \mathrm{~nm}(200 \mathrm{~K})$, with typically around $50 \%$ of the creep already occurring during the first $15 \mathrm{~ms}$. We thus conclude that creep of the tip can affect the aging behavior only during the first milliseconds of the hold time, but can be neglected for extended hold times.

\section{APPENDIX C: MD SIMULATION DETAILS}

The MD simulations were carried out using LAMMPS [55]. The equations of motion are integrated with velocityVerlet algorithm [56] with a time step of $0.001 \mathrm{ps,} \mathrm{which}$ correctly samples the dynamics of the lightest atoms, i.e., oxygen, at high temperatures or pressures [57,58]. The coordinates were saved every picosecond. The temperature and pressure are regulated via a Nose-Hoover [59-62] thermostat and barostat, respectively. In the preparatory annealing-quenching cycles, NPT (constant temperature 
and pressure) ensemble simulations were performed, whereas all other simulations were performed in the $N V T$ (constant volume and temperature) ensemble.

\section{APPENDIX D: $\mathrm{SiO}_{2}$ ATOMIC MODELS (FORCE FIELDS) AND THEIR SUITABILITY FOR INDENTATION STUDIES}

$\mathrm{Si}-\mathrm{Si}, \mathrm{Si}-\mathrm{O}, \mathrm{O}-\mathrm{O}$ interactions were modeled via a bond-order three-body Tersoff $[63,64]$ potential parametrized by Munetoh et al. [63] to describe a $\mathrm{Si}, \mathrm{O}$ mixed system. Munetoh [63] parametrization is based on experimental data of $\alpha$-quartz as well as ab initio calculations of small fragments. This parametrization provided results in excellent agreement with experimental data [63]: namely, the structural properties and energies of the various crystalline phases as well as the amorphous phase for silica $[57,63]$, radial distribution functions, and phonon density of states of silica glass [63]. Furthermore, it has also been experimentally validated to describe atomic rearrangements in silica glass surfaces induced using aberration-corrected transmission electron microscopy [37], transformation mechanisms [65] and thermal conductivity $[65,66]$ in porous silica, and also the Hugoniot curves $[58,67]$ describing the dynamic shock-induced response of silica. Additionally, a good agreement with experimental results has also been found in other mechanical properties such as the deformation behavior of amorphous silica upon indentation [40,41].

\section{APPENDIX E: SIMULATION PROTOCOL}

The simulation protocol was composed by two stages: a preparatory stage, where we generate the initial coordinates of the tip and the surface, and a second stage, where we indent the surface with a constant normal load and temperature. In the first stage, we built an amorphous $\mathrm{SiO}_{2}$ surface of $60 \times 60 \times 18 \mathrm{~nm}^{3}$ composed by 3345408 atoms by using the atomic configurations of amorphous $\mathrm{SiO}_{2}$ cubes of $(5)^{3} \mathrm{~nm}^{3}$ reported in Ref. [68]. Then, in order to remove the superperiodicity of $5 \mathrm{~nm}$, we improved $\mathrm{SiO}_{2}$ amorphization by following the standard melting-quenching procedure $[2,12,13,40,41,57,58,63,65-67,69]$. This protocol consisted of melting $\mathrm{SiO}_{2}$ by heating it up to $5000 \mathrm{~K}$ in $250 \mathrm{ps}$ at zero pressure followed by a quenching to $300 \mathrm{~K}$ with a cooling rate of $11 \mathrm{~K} / \mathrm{ps}$. Note that during the annealing stage the temperature was raised up to $5000 \mathrm{~K}$, which is well above the melting point of $\mathrm{SiO}_{2}$ obtained experimentally [70]. Although already at temperatures of 1900-2400 K SiO 2 started to melt, in agreement with previous works [57], annealing to higher temperature promotes a sufficiently fast diffusion of the atoms so that we could produce an equilibrated liquid in the short time span (as compared to timescales used in the experiments) of the simulation. Additionally, as in Ref. [2], by using freeboundary conditions in the out-of-plane direction, the obtained surface displays a roughness on the top and bottom part of the surface. Moreover, the surface stress of the asprepared surface is allowed to relax during the $\sim 0.5 \mathrm{~ns}$ quenching stage. Then, we extract a slab of $25 \times 25 \mathrm{~nm}^{2}$ and a thickness of $8.5 \mathrm{~nm}$. Similarly, we obtain the tip atomic coordinates by selecting all atoms within a sphere with a radius of $10.25 \mathrm{~nm}$ and then we kept only the bottommost atoms $8.7 \mathrm{~nm}$. In order to avoid any synchronization on the roughness of the surface and the tip, the tip roughness is provided through the bottom part of the larger slab, while the roughness of the surface is provided through the top part of the larger slab. In total, this system composed by the tip and surface contains a total of 352137 atoms and the charge neutrality was assured throughout the whole process by imposing the ratio $\mathrm{SiO}_{2}$. The density of the amorphous silica thus created is of $2.22 \mathrm{~g} / \mathrm{cm}^{3}$, in agreement with the experimental value [71] of $2.20 \mathrm{~g} / \mathrm{cm}^{3}$ and previous MD simulations $[40,41,57,58,63,65-67,69,71]$. The amorphization of the sample has been confirmed by calculating the radial distribution functions of $\mathrm{Si}-\mathrm{Si}, \mathrm{Si}-\mathrm{O}$, and $\mathrm{O}-\mathrm{O}$ pairs whose first peaks occur, respectively, at 3.13, 1.65 and $2.67 \AA$, in agreement with previous MD simulations as well as with experimental results [40,41,57,58,63,65-67,69,71]. The root-mean-square roughness of the surface thus obtained is of $1.56 \AA$, which is similar to a previously reported MD simulation whose protocol was followed here [2] and close to the experimental value [45]. In the second stage, the position of the bottom atoms of the surface (bottom $0.7 \mathrm{~nm}$, shown in green in Fig. 1) is kept fixed during the indentation process. Initially the tip is brought into contact with the surface while assuring no atomic bonds exist at $t=0$, and then the indentation starts by applying a constant normal force of $40 \mathrm{nN}$ (with the exception of the 80-nN simulation) on the tip's top atoms (top $0.7 \mathrm{~nm}$, shown in green in Fig. 1). All the other atoms are allowed to move freely during the indentation process. When the contact is formed we follow the number of bonds formed between the tip and the surface atoms. The criteria for stable bond formation at the interface correspond to $\mathrm{Si}-\mathrm{O}$ or $\mathrm{O}-\mathrm{O}$ pairs of atoms that are within the maximum equilibrium distance reached due to solely thermal fluctuations at room temperature, i.e., 1.7 and $1.55 \AA$, respectively. Additionally, these distances are close to the average distance defined in the force field $[57,63]$ and also agree well with the experimental distances of these bonds [74]. By recording the coordinates of all atoms every picosecond, we then compute the average number of bonds between the tip and the surface every $50 \mathrm{ps}$. The results thus obtained are shown in Fig. 3.

[1] B. Cotterell and J. Kamminga, Mechanics of Pre-industrial Technology (Cambridge University Press, Cambridge, England, 1992).

[2] S. Li, Q. Li, R. W. Carpick, P. Gumbsch, X. Z. Liu, X. Ding, J. Sun, and J. Li, The Evolving Quality of Frictional Contact with Graphene, Nature (London) 539, 541 (2016). 
[3] Q. Li, T. E. Tullis, D. Goldsby, and R. W. Carpick, Frictional Ageing from Interfacial Bonding and the Origins of Rate and State Friction, Nature (London) 480, 233 (2011).

[4] N. Manini, G. Mistura, G. Paolicelli, E. Tosatti, and A. Vanossi, Current Trends in the Physics of Nanoscale Friction, Adv. Phys. X 2, 569 (2017).

[5] S. Y. Krylov and J. W. Frenken, The Physics of Atomic-Scale Friction: Basic Considerations and Open Questions, Phys. Status Solidi B 251, 711 (2014).

[6] A. Vanossi, N. Manini, M. Urbakh, S. Zapperi, and E. Tosatti, Colloquium: Modeling Friction: From Nanoscale to Mesoscale, Rev. Mod. Phys. 85, 529 (2013).

[7] M. Evstigneev, A. Schirmeisen, L. Jansen, H. Fuchs, and P. Reimann, Contact Ageing in Atomic Friction, J. Phys. Condens. Matter 20, 354001 (2008).

[8] N. N. Gosvami, M. Feldmann, J. Peguiron, M. Moseler, A. Schirmeisen, and R. Bennewitz, Ageing of a Microscopic Sliding Gold Contact at Low Temperatures, Phys. Rev. Lett. 107, 144303 (2011).

[9] J. Rottler and M. O. Robbins, Unified Description of Aging and Rate Effects in Yield of Glassy Solids, Phys. Rev. Lett. 95, 225504 (2005).

[10] F. P. Bowden and D. Tabor, The Area of Contact between Stationary and between Moving Surfaces, Proc. R. Soc. A 169, 391 (1939).

[11] J. Greenwood and J. Williamson, Contact of Nominally Flat Surfaces, Proc. R. Soc. A 295, 300 (1966).

[12] Y. Liu and I. Szlufarska, Chemical Origins of Frictional Aging, Phys. Rev. Lett. 109, 186102 (2012).

[13] A. Li, Y. Liu, and I. Szlufarska, Effects of Interfacial Bonding on Friction and Wear at Silica/Silica Interfaces, Tribol. Lett. 56, 481 (2014).

[14] K. Tian, N. N. Gosvami, D. L. Goldsby, Y. Liu, I. Szlufarska, and R. W. Carpick, Load and Time Dependence of Interfacial Chemical Bond-Induced Friction at the Nanoscale, Phys. Rev. Lett. 118, 076103 (2017).

[15] I. Barel, M. Urbakh, L. Jansen, and A. Schirmeisen, Multibond Dynamics of Nanoscale Friction: The Role of Temperature, Phys. Rev. Lett. 104, 066104 (2010).

[16] I. Barel, M. Urbakh, L. Jansen, and A. Schirmeisen, Unexpected Temperature and Velocity Dependencies of Atomic-Scale Stick-Slip Friction, Phys. Rev. B 84, 115417 (2011).

[17] C. Ganser, C. Czibula, D. Tscharnuter, T. Schöberl, C. Teichert, and U. Hirn, Combining Adhesive Contact Mechanics with a Viscoelastic Material Model to Probe Local Material Properties by AFM, Soft Matter 14, 140 (2018).

[18] E. Riedo, F. Levy, and H. Brune, Kinetics of Capillary Condensation in Nanoscopic Sliding Friction, Phys. Rev. Lett. 88, 185505 (2002).

[19] R. Szoszkiewicz and E. Riedo, Nucleation Time of Nanoscale Water Bridges, Phys. Rev. Lett. 95, 135502 (2005).

[20] J. J. Mazo, D. Dietzel, A. Schirmeisen, J. G. Vilhena, and E. Gnecco, Time Strengthening of Crystal Nanocontacts, Phys. Rev. Lett. 118, 246101 (2017).

[21] T. Baumberger and C. Caroli, Solid Friction from Stick-Slip Down to Pinning and Aging, Adv. Phys. 55, 279 (2006).

[22] A. Ploessl, Wafer Direct Bonding: Tailoring Adhesion between Brittle Materials, Mater. Sci. Eng. Rep. 25, 1 (1999).
[23] G. Vigil, Z. Xu, S. Steinberg, and J. Israelachvili, Interactions of Silica Surfaces, J. Colloid Interface Sci. 165, 367 (1994).

[24] T. A. Michalske and E. Fuller, Closure and Repropagation of Healed Cracks in Silicate Glass, J. Am. Ceram. Soc. 68, 586 (1985).

[25] J. H. Dieterich and B. D. Kilgore, Direct Observation of Frictional Contacts: New Insights for State-Dependent Properties, Pure Appl. Geophys. 143, 283 (1994).

[26] M. Feldmann, D. Dietzel, H. Fuchs, and A. Schirmeisen, Influence of Contact Aging on Nanoparticle Friction Kinetics, Phys. Rev. Lett. 112, 155503 (2014).

[27] C. Petzold, M. Koch, and R. Bennewitz, Friction Force Microscopy of Tribochemistry and Interfacial Ageing for the $\mathrm{SiO}_{x} / \mathrm{Si} / \mathrm{Au}$ System, Beilstein J. Nanotechnol. 9, 1647 (2018).

[28] C. M. Mate, G. M. McClelland, R. Erlandsson, and S. Chiang, Atomic-Scale Friction of a Tungsten Tip on a Graphite Surface, Phys. Rev. Lett. 59, 1942 (1987).

[29] K. M. Frye and C. Marone, Effect of Humidity on Granular Friction at Room Temperature, J. Geophys. Res. 107, ETG 11-1 (2002).

[30] L. Prandtl, Ein Gedankenmodell zur Kinetischen Theorie der Festen Koerper, Z. Angew. Math. Mech. 8, 85 (1928).

[31] V. L. Popov and J. A. T. Gray, Prandtl-Tomlinson Model: History and Applications in Friction, Plasticity, and Nanotechnologies, Z. Angew. Math. Mech. 92, 683 (2012).

[32] L. Jansen, H. Hoelscher, H. Fuchs, and A. Schirmeisen, Temperature Dependence of Atomic-Scale Stick-Slip Friction, Phys. Rev. Lett. 104, 256101 (2010).

[33] E. Gnecco, R. Bennewitz, T. Gyalog, C. Loppacher, M. Bammerlin, E. Meyer, and H.J. Güntherodt, Velocity Dependence of Atomic Friction, Phys. Rev. Lett. 84, 1172 (2000).

[34] A. Socoliuc, R. Bennewitz, E. Gnecco, and E. Meyer, Transition from Stick-Slip to Continuous Sliding in Atomic Friction: Entering a New Regime of Ultralow Friction, Phys. Rev. Lett. 92, 134301 (2004).

[35] M. Evstigneev and P. Reimann, Thermally Activated Contact Strengthening Explains Nonmonotonic Temperature and Velocity Dependence of Atomic Friction, Phys. Rev. X 3, 041020 (2013).

[36] Y. Liu and I. Szlufarska, Chemical Origins of Frictional Aging, Phys. Rev. Lett. 109, 186102 (2012).

[37] P. Y. Huang, S. Kurasch, J. S. Alden, A. Shekhawat, A. A. Alemi, P. L. McEuen, J.P. Sethna, U. Kaiser, and D. A. Muller, Imaging Atomic Rearrangements in Two-Dimensional Silica Glass: Watching Silica's Dance, Science 342, 224 (2013).

[38] A. J. Lunt, P. Chater, and A. M. Korsunsky, On the Origins of Strain Inhomogeneity in Amorphous Materials, Sci. Rep. 8, 1574 (2018).

[39] M. Murakami, S. Kohara, N. Kitamura, J. Akola, H. Inoue, A. Hirata, Y. Hiraoka, Y. Onodera, I. Obayashi, J. Kalikka, N. Hirao, T. Musso, A. S. Foster, Y. Idemoto, O. Sakata, and Y. Ohishi, Ultrahigh-Pressure Form of $\mathrm{SiO}_{2}$ Glass with Dense Pyrite-Type Crystalline Homology, Phys. Rev. B 99, 045153 (2019).

[40] J. Chen, J. Shi, Y. Wang, J. Sun, J. Han, K. Sun, and L. Fang, Nanoindentation and Deformation Behaviors of Silicon 
Covered with Amorphous $\mathrm{SiO}_{2}$ : A Molecular Dynamic Study, RSC Adv. 8, 12597 (2018).

[41] J. Chen, J. Shi, M. Zhang, W. Peng, L. Fang, K. Sun, and J. Han, Effect of Indentation Speed on Deformation Behaviors of Surface Modified Silicon: A Molecular Dynamics Study, Comput. Mater. Sci. 155, 1 (2018).

[42] J. Chen, J. Shi, Z. Chen, M. Zhang, W. Peng, L. Fang, K. Sun, and J. Han, Mechanical Properties and Deformation Behaviors of Surface-Modified Silicon: A Molecular Dynamics Study, J. Mater. Sci. 54, 3096 (2019).

[43] W. J. Malfait, W. E. Halter, and R. Verel, ${ }^{29} \mathrm{Si} N M R$ Spectroscopy of Silica Glass: $\mathrm{T}_{1}$ Relaxation and Constraints on the Si-O-Si Bond Angle Distribution, Chem. Geol. 256, 269 (2008).

[44] E. Lascaris, M. Hemmati, S. V. Buldyrev, H. E. Stanley, and C. A. Angell, Search for a Liquid-Liquid Critical Point in Models of Silica, J. Chem. Phys. 140, 224502 (2014).

[45] F. Giannazzo, S. Sonde, R. L. Nigro, E. Rimini, and V. Raineri, Mapping the Density of Scattering Centers Limiting the Electron Mean Free Path in Graphene, Nano Lett. 11, 4612 (2011).

[46] Q. Li, Y. Dong, D. Perez, A. Martini, and R. W. Carpick, Speed Dependence of Atomic Stick-Slip Friction in Optimally Matched Experiments and Molecular Dynamics Simulations, Phys. Rev. Lett. 106, 126101 (2011).

[47] K. Tian, N. N. Gosvami, D. L. Goldsby, Y. Liu, I. Szlufarska, and R. W. Carpick, Load and Time Dependence of Interfacial Chemical Bond-Induced Friction at the Nanoscale, Phys. Rev. Lett. 118, 076103 (2017).

[48] J. G. Vilhena, C. Pimentel, P. Pedraz, F. Luo, P. A. Serena, C. M. Pina, E. Gnecco, and R. Prez, Atomic-Scale Sliding Friction on Graphene in Water, ACS Nano 10, 4288 (2016).

[49] H. Eyring, Viscosity, Plasticity, and Diffusion as Examples of Absolute Reaction Rates, J. Chem. Phys. 4, 283 (1936).

[50] B. Gotsmann and M. A. Lantz, Atomistic Wear in a Single Asperity Sliding Contact, Phys. Rev. Lett. 101, 125501 (2008).

[51] B. Weber, T. Suhina, A. M. Brouwer, and D. Bonn, Frictional Weakening of Slip Interfaces, Sci. Adv. 5, eaav7603 (2019).

[52] T. Suhina, B. Weber, C. E. Carpentier, K. Lorincz, P. Schall, D. Bonn, and A. M. Brouwer, Fluorescence Microscopy Visualization of Contacts between Objects, Angew. Chem., Int. Ed. Engl. 54, 3688 (2015).

[53] B. Weber, T. Suhina, T. Junge, L. Pastewka, A. M. Brouwer, and D. Bonn, Molecular Probes Reveal Deviations from Amontons' Law in Multi-Asperity Frictional Contacts, Nat. Commun. 9, 1 (2018).

[54] P. Bilas, L. Romana, B. Kraus, Y. Bercion, and J. L. Mansot, Quantitative Characterization of Friction Coefficient Using Lateral Force Microscope in the Wearless Regime, Rev. Sci. Instrum. 75, 415 (2004).

[55] S. Plimpton, Fast Parallel Algorithms for Short-Range Molecular Dynamics, J. Comput. Phys. 117, 1 (1995).

[56] D. Beeman, Some Multistep Methods for Use in Molecular Dynamics Calculations, J. Comput. Phys. 20, 130 (1976).

[57] S. K. D. Nath, Study of the Effect of Sizes on the Structural Properties of $\mathrm{SiO}_{2}$ Glass by Molecular Dynamics Simulations, J. Non-Cryst. Solids 376, 50 (2013).
[58] J. Wang, A. M. Rajendran, and A. M. Dongare, Atomic Scale Modeling of Shock Response of Fused Silica and $\alpha$-Quartz, J. Mater. Sci. 50, 8128 (2015).

[59] W. Shinoda, M. Shiga, and M. Mikami, Rapid Estimation of Elastic Constants by Molecular Dynamics Simulation under Constant Stress, Phys. Rev. B 69, 134103 (2004).

[60] G. J. Martyna, Constant Pressure Molecular Dynamics Algorithms, J. Chem. Phys. 101, 4177 (1994).

[61] M. Parrinello and A. Rahman, Polymorphic Transitions in Single Crystals: A New Molecular Dynamics Method, J. Appl. Phys. 52, 7182 (1981).

[62] M. Tuckerman, J. Alejandre, R. López-Rendón, A. Jochim, and G. Martyna, A Liouville-Operator Derived MeasurePreserving Integrator for Molecular Dynamics Simulations in the Isothermal-Isobaric Ensemble, J. Phys. A 39, 5629 (2006).

[63] S. Munetoh, T. Motooka, K. Moriguchi, and A. Shintani, Interatomic Potential for Si-O Systems Using Tersoff Parameterization, Comput. Mater. Sci. 39, 334 (2007).

[64] J. Tersoff, Modeling Solid-State Chemistry: Interatomic Potentials for Multicomponent Systems, Phys. Rev. B 39, 5566 (1989).

[65] S. Zhang, M. Perez-Page, K. Guan, E. Yu, J. Tringe, R. H. Castro, R. Faller, and P. Stroeve, Response to Extreme Temperatures of Mesoporous Silica MCM-41: Porous Structure Transformation Simulation and Modification of Gas Adsorption Properties, Langmuir 32, 11422 (2016).

[66] W. Zhu, G. Zheng, S. Cao, and H. He, Thermal Conductivity of Amorphous $\mathrm{SiO}_{2}$ Thin Film: A Molecular Dynamics Study, Sci. Rep. 8, 10537 (2018).

[67] R. Su, M. Xiang, J. Chen, S. Jiang, and H. Wei, Molecular Dynamics Simulation of Shock Induced Ejection on Fused Silica Surface, J. Appl. Phys. 115, 193508 (2014).

[68] E. R. Cruz-Chu, A. Aksimentiev, and K. Schulten, Water Silica Force Field for Simulating Nanodevices, J. Phys. Chem. B 110, 21497 (2006).

[69] J. Wang, A. M. Rajendran, and A. M. Dongare, Atomic Scale Modeling of Shock Response of Fused Silica and $\alpha$-Quartz, J. Mater. Sci. 50, 8128 (2015).

[70] E. Ringdalen and M. Tangstad, Softening and Melting of $\mathrm{SiO}_{2}$, An Important Parameter for Reactions with Quartz in Si Production, in Advances in Molten Slags, Fluxes, and Salts: Proceedings of the 10th International Conference on Molten Slags, Fluxes and Salts 2016, edited by R. G. Reddy, P. Chaubal, P. C. Pistorius, U. Pal (Springer, Cham, 2016), p. 43.

[71] F. Yuan and L. Huang, Molecular Dynamics Simulation of Amorphous Silica under Uniaxial Tension: From Bulk to Nanowire, J. Non-Cryst. Solids 358, 3481 (2012).

[72] S. Li, Q. Li, R. W. Carpick, P. Gumbsch, X.Z. Liu, X. Ding, J. Sun, and J. Li, The Evolving Quality of Frictional Contact with Graphene, Nature (London) 539, 541 (2016).

[73] S. Munetoh, T. Motooka, K. Moriguchi, and A. Shintani, Interatomic Potential for SiO Systems using Tersoff Parameterization, Comput. Mater. Sci. 39, 334 (2007).

[74] W. Haynes, CRC Handbook of Chemistry and Physics, 100 Key Points, 96th ed. (CRC Press, Boca Raton, FL, 2015). 